\title{
Improving Mobile and Ad-hoc Networks performance using Group-Based Topologies
}

\author{
Jaime Lloret $^{1}$, Miguel Garcia ${ }^{2}$ and Jesus Tomas ${ }^{3}$ \\ Department of Communications \\ Polytechnic University of Valencia \\ Camino Vera s/n, 46022, Valencia (Spain) \\ ${ }^{1}$ jlloret@dcom.upv.es, ${ }^{2}$ migarpi@posgrado.upv.es, ${ }^{3}$ jtomas@dcom.upv.es
}

\begin{abstract}
Many works related with mobile and ad-hoc networks routing protocols present new proposals with better or enhanced features, others just compare them or present an application environment, but this work tries to give another point of view. Why don't we see the network as a whole and split it intro groups to give better performance to the network regardless of the used routing protocol?. First, we will demonstrate, through simulations, that grouping nodes in a mobile and ad-hoc networks improves the whole network by diminishing the average network delay and also the routing traffic received by the nodes. Then, we will show which one of the actual fully standardized protocols (DSR [1], AODV [2] and OLSR [3]) gives better performance to the whole network when there are groups of nodes. This paper starts a new research line and urges the researchers to think on it and design group-based protocols.
\end{abstract}

Keywords: MANET, group-based topologies, network performance.

\section{Introduction}

The routing protocols in mobile and ad-hoc networks are divided into three types: proactive (which update the routing tables of all the nodes periodically), reactive (which maintain routing routes in their tables only when a node has to communicate with another node in the network) and hybrid (which are a combination of the other two types, taking the advantages of both types). There are many works in the literature that compare the performance of the routing protocols. The most compared protocols have been DSR and AODV. In references [4] and [5] we can see their comparison taking into account some parameters such as the packet delivery fraction, the average delay, the normalized routing load and the throughput consumed when the network load, the mobility and the network size vary. The work in reference [6] added the STAR protocol to the comparison and they measured the data delivery, the control overhead and the data latency. Reference [7] compared DSR and AODV with DSDV taking into account the average delay, the throughput and the control overhead with varied mobility. On the other hand, reference [8] compared DSR, AODV and TORA to analyze the control traffic sent, the data traffic received, the data traffic sent, the throughput, the retransmission attempts, the radio receiver throughput, the radio 
receiver utilization, the average power, the radio transmitter utilization, the radio transmitter throughput, routing traffic received, routing traffic sent, number of hops and route discovery time. The paper in reference [9] compares the number of packets sent and the traffic sent by DSDV, TORA, DSR and AODV protocols in networks of 50 mobile nodes. Other works compared 5 protocols such as the one presented in [10], where AODV, PAODV, CBRP, DSR and DSDV were compared taking into account the data packet throughput, the average data packet delay and the normalized packet overhead for various number of traffic sources.

Current IETF standardized protocols are AODV [1], DSR [2] and OLSR [3]. None of the works aforementioned have compared them from the group-based topology point of view. We are going to analyze and study their performance when there are group of nodes in their topology.

A cluster is made by a cluster head node, cluster gateways and cluster members. The cluster head node is the parent node of the cluster, which manages and checks the status of the links in the cluster, and routes the information to the right clusters. The rest of the nodes in a cluster are all leaf nodes. The size of the cluster is usually about 1 or 2 hops from the cluster head node. Cluster-based networks are a subset of the group-based networks, because every cluster could be considered as a group. But a group-based network is capable of having any type of topology inside the group, not only clusters. We will take care of group-based topologies in this paper.

The paper is structured as follows. Section 2 shows and analyzes the differences between DSR, AODV and OLSR protocols when regular and group-based topologies are used. The group-based topologies comparison is shown in section 3. Finally, section 4 gives our conclusions.

\section{Group-based topology performance}

\subsection{Test Bench}

This sub-section presents the test-bench used for all the evaluated protocols. The number of nodes and the coverage area of the network have been varied. Each protocol has been simulated in 4 scenarios: (1) With fixed nodes, (2) With mobile nodes and failures, (3) With grouped nodes and (4) With grouped mobile nodes and failures. Each scenario has been simulated for 100 and 250 nodes, to observe the system scalability. Instead of a standard structure we have chosen a random topology. Figure 1 shows the 100 nodes topology (in a 750x750 $\mathrm{m}^{2}$ area) and Figure 2 shows the 250 nodes topology (in a $1 \mathrm{Km}^{2}$ area). It has been obtained using the version Modeler of OPNET simulator [11]. Both topologies have been created using different seeds. Arrows indicate that nodes are mobile and change their position constantly. The green lines from each node (blue circles) indicate the node mobility. We can see that the nodes are inside a blue box. This box shows a wireless area and it has been used to delimit the mobility area of the nodes. In that area, a node can move randomly during the simulation. The physical topology doesn't follow any known pattern. The obtained data don't depend on the initial topology of the nodes or on their movement pattern, because all of it has been fortuitous. 


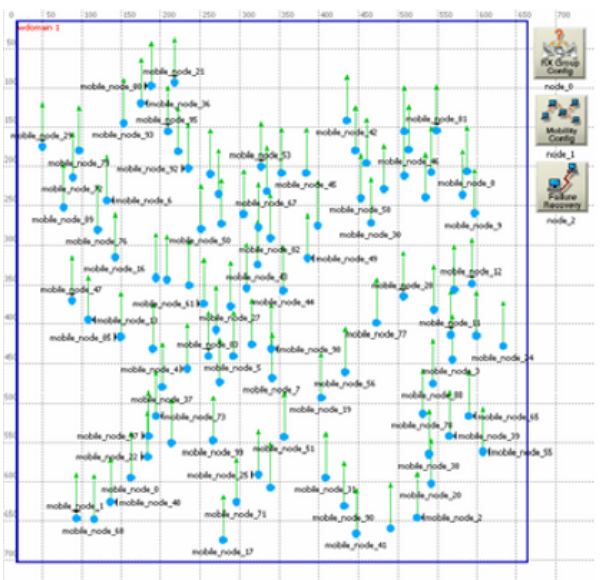

Fig 1. Topology with 100 nodes.

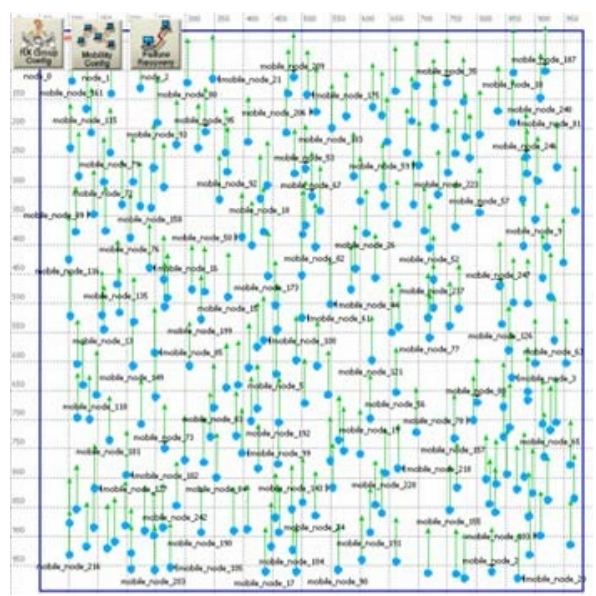

Fig 2. Topology with 250 nodes.

We have created 6 groups for the 100 nodes topology, covering approximately, a circular area with a 150 meter radius each group. There are 16 or 17 nodes approximately, in each group. The number of nodes in each group varies because of the node's random mobility. A node can change a group anytime. For the 250 nodes topology, we have created 12 groups, with 15 or 16 nodes per group approximately covering a circular area with a 150 meter radius each group.

The ad-hoc nodes of the topologies have a $40 \mathrm{MHz}$ processor, a $512 \mathrm{~KB}$ memory card, a radio channel of $1 \mathrm{Mbps}$ and their working frequency is $2.4 \mathrm{GHz}$. Their maximum coverage radius is 50 meters. This is a conservative value because most of the nodes in ad-hoc network have larger coverage radius, but we preferred to have lower transmitting power for the ad-hoc devices to enlarge their time of life.

We have forced node failures at $t=200 \mathrm{sec}$., $\mathrm{t}=400 \mathrm{sec}$. and $\mathrm{t}=1200 \mathrm{sec}$. in each network, with a recovering process of $300 \mathrm{sec}$., to take measurements from the mobile nodes simulation when the physical topology changes.

The MANET traffic generated by OPNET has been used as the simulations' traffic load. We inject this traffic 100 seconds after the simulation starts. We have configured the traffic arrival with a Poisson distribution (with a mean time between arrivals of 30 seconds). The packet size follows an exponential distribution with a mean value of 1024 bits. The destination address of the injected traffic is random to obtain a simulation independent of the traffic direction. We have simulated the four scenarios for DSR, AODV and OLSR protocols. The results obtained are shown in the following sub-sections.

\subsection{DSR, AODV and OLSR in group-based topologies}

Figures 3 and 4 show the average delay of the DSR protocol in fixed and mobile topologies at the application layer. In figure 3 we observe that group-based topologies have an average delay close to 0.005 seconds regardless of the number of nodes in the network. In the regular network the delay has a value of 0.02 seconds for 100 -nodes topology and of 0.03 seconds for the 250-nodes topology when the network 
converges. In the case of the 100-nodes topology there is an improvement of $75 \%$ and it is better in the 250-nodes topology (an 83\% of improvement). The topologies with mobility and errors (figure 4) shows that the average delays at the application layer are higher in the group-based topologies till the network converges. Although groupbased topologies present worse behaviour till 1300 seconds, when the network is stabilized, group-based topologies have an improvement around 5\%.

Then, we have compared the routing traffic received in the DSR protocol (figures 5 and 6). Figure 5 shows that the traffic is quite stable due to the characteristics of the network. It is due to it is a fixed network without errors and failures. The traffic received in the 250-node topology is around $500 \mathrm{Kbits} / \mathrm{s}$, but when we group the nodes this traffic decreases until $200 \mathrm{Kbits} / \mathrm{s}$ (a 60\% of improvement). The value obtained in a 100-node topology $(250 \mathrm{Kbits} / \mathrm{s})$, is also improved when we group the nodes $(100 \mathrm{Kbits} / \mathrm{s})$, therefore there is a $60 \%$ of improvement. In figure 6 we observe a similar behaviour. In this case we conclude that when there are errors and failures in the 250-nodes topology the traffic fluctuates and is less stable (we can observe it in the intervals from 600 to 800 seconds and around 1200 seconds). We also observe that the instability is much lower in group-based topologies. 100-nodes topology has a mean value around $175 \mathrm{Kbits} / \mathrm{s}$, while 100-nodes group-based topology has a mean value around $95 \mathrm{Kbits} / \mathrm{s}$, so there is an improvement of $46 \%$. On the other hand, 250nodes topology has a mean value around $400 \mathrm{Kbits} / \mathrm{s}$, while 250 -nodes group-based topology has a mean value around $180 \mathrm{Kbits} / \mathrm{s}$, so there is an improvement of $55 \%$.

The average delay at the application layer in the AODV protocol can be seen in figures 7 and 8. Both topologies, 100-nodes and 250-nodes, give an average delay higher than 0.5 seconds when the network converges, but there are some peaks higher than 2.5 seconds. On the other hand, group-based topologies have a similar delay which is around 0.15 seconds. Group-based topologies improve the delay at the application layer in $70 \%$. When the topology with mobile nodes is used, the simulation shown in figure 8 is obtained. In case of 250 nodes, there is a delay of 1 second when the network has converged. The case of 100 nodes gives an average delay of 0.75 seconds approximately. When there are group-based topologies, the delay decreases to 0.25 seconds in both cases. There is an improvement of $75 \%$ for the 250 -nodes topology and $67 \%$ for the 100 -nodes topology.

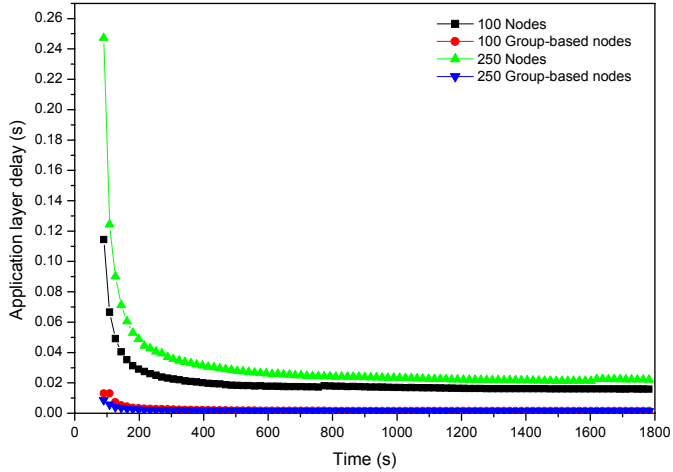

Fig. 3. DSR average delay at the application layer in fixed topologies.

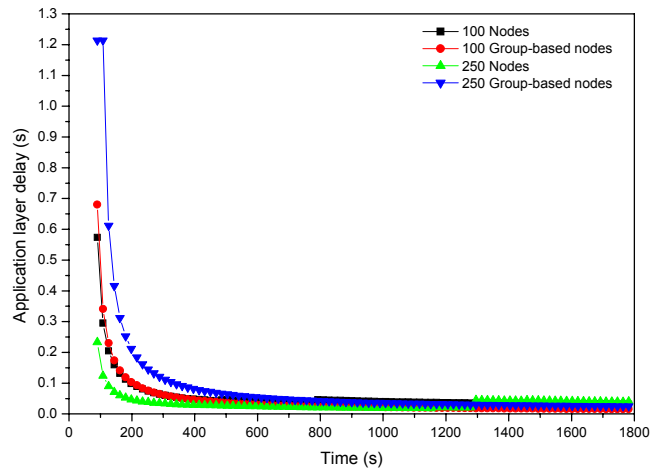

Fig. 4. DSR average delay at the application layer in mobile topologies. 


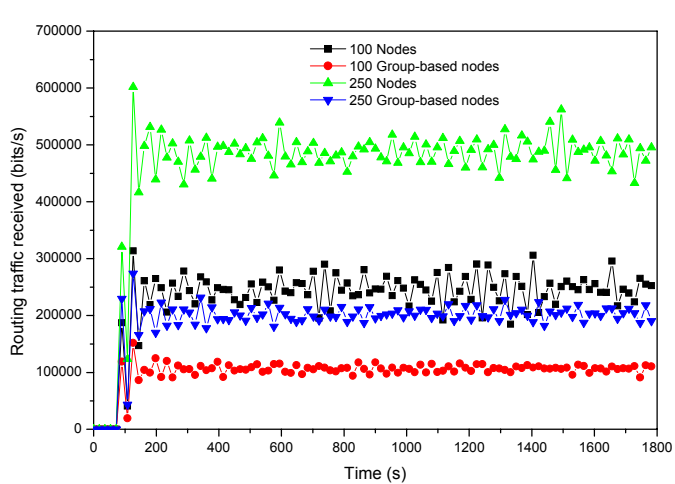

Fig. 5. DSR routing traffic received in fixed topologies.

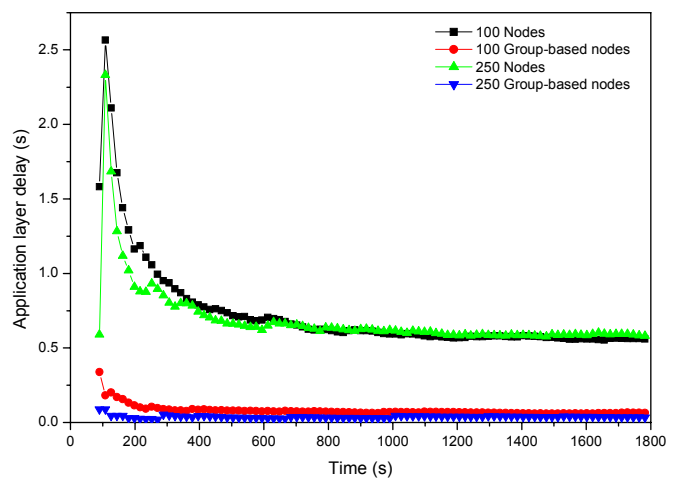

Fig. 7. AODV average delay at the application layer in fixed topologies.

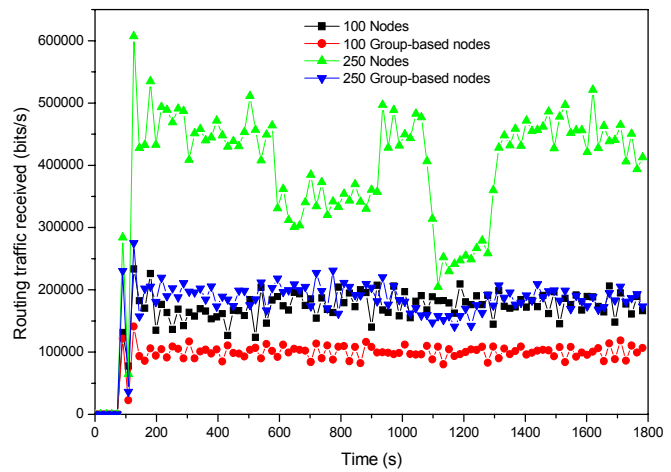

Fig. 6. DSR routing traffic received in mobile topologies.

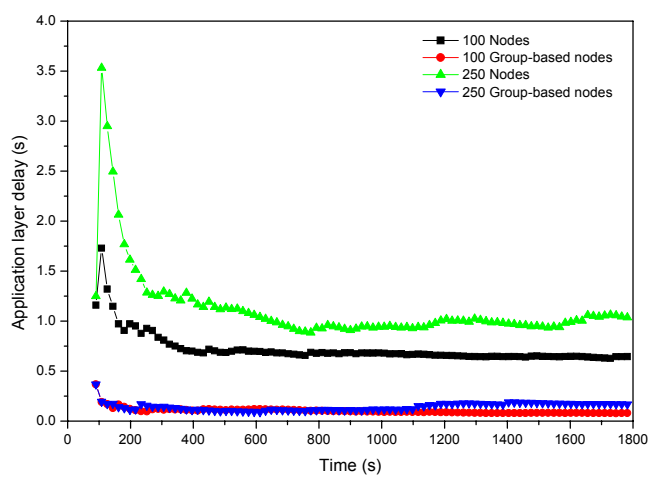

Fig. 8. AODV average delay at the application layer in mobile topologies.

The routing traffic received for the AODV in each simulated topology can be seen in figures 9 and 10. We observe that the routing traffic received is independent of the mobility of the nodes. In figure 9 we can see that the routing traffic goes from 440 $\mathrm{Kbits} / \mathrm{s}$ for 250 -node case to $250 \mathrm{Kbits} / \mathrm{s}$ when there are group of nodes (a $43 \%$ of improvement). In the 100 -node case, it goes from $230 \mathrm{Kbits} / \mathrm{s}$ to $140 \mathrm{Kbits} / \mathrm{s}$ when it is a group-based topology (a 39\% of improvement). When there are mobility and errors and failures (see figure 10), in the 250-node topology the values go from 440 $\mathrm{Kbits} / \mathrm{s}$ to $250 \mathrm{Kbits} / \mathrm{s}$ in the group-based topology (a $43 \%$ of improvement). We obtained $200 \mathrm{Kbits} / \mathrm{s}$ in the regular 100-node topology and $135 \mathrm{Kbits} / \mathrm{s}$ for the groupbased one (a $32 \%$ of improvement).

In figure 11, the delay at the application layer simulated for the OLSR protocol using fixed topologies is shown. In the case of 250 nodes we have obtained a delay around 0.015 seconds, which has changed to 0.0035 seconds in the case of 250 -nodes group-based topology (there is a $76 \%$ of improvement). In the case of 100 nodes, it has decreased from 0.005 seconds in the regular topology to 0.002 seconds in the group-based topology, so there is a $60 \%$ of improvement. When there is mobility and 
errors and failures in the network for the OLSR protocol (see figure 12), we observe that the 100-nodes regular topology has a delay at the application layer of 0.007 seconds when the network has converged, but there is a delay of 0.0025 seconds for the 100-nodes group-based topology (a 64\% of improvement). In the case of 250 nodes the improvement is around $60 \%$. We have obtained a delay of 0.005 seconds in the regular topology versus 0.002 seconds in the group-based topology.

Finally, we have studied the behaviour of the OLSR protocol analyzing the mean routing traffic received (figures 13 and 14). The routing traffic received in the 100node fixed topology was around $180 \mathrm{Kbits} / \mathrm{s}$, while in group-based topology has decreased to $70 \mathrm{Kbits} / \mathrm{s}$, so there is a $61 \%$ of improvement. In the 250 -node topology case, we appreciate that this traffic was approximately $300 \mathrm{Kbits} / \mathrm{s}$, but there are values lower than $150 \mathrm{Kbits} / \mathrm{s}$ in the group-based topology (figure 13). So there is a $50 \%$ of improvement. Figure 14 shows the results of a network with mobility and errors and failures. We have observed some fluctuations due to the failures and errors in the network, in both 100-node and 250-node topologies. Those fluctuations are minimized when we use group-based topologies. Improvements of $61 \%$ and $50 \%$ are obtained in 100-node and 250-node topologies, respectively.

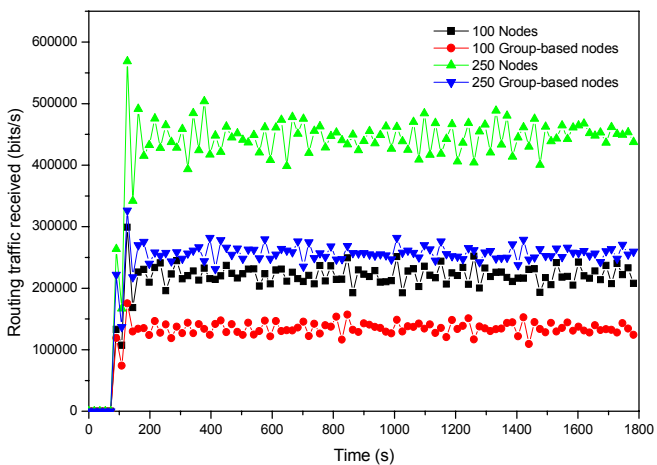

Fig. 9. AODV routing traffic received in fixed topologies.

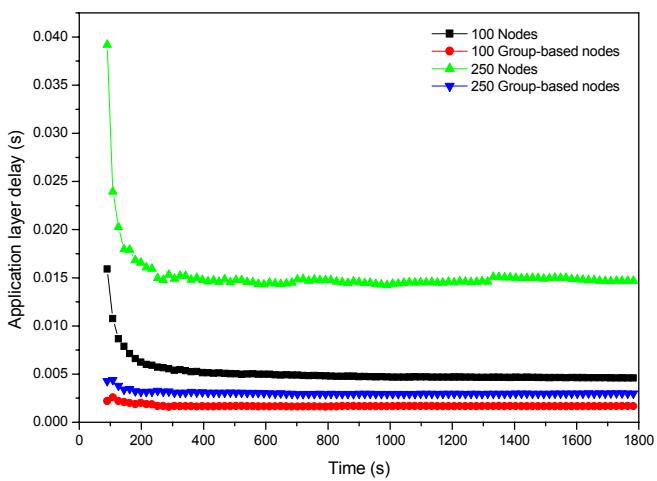

Fig. 11. OLSR average delay at the application layer in fixed topologies.

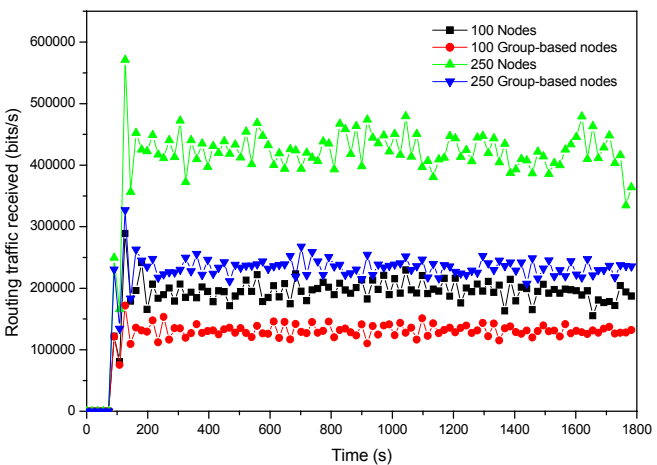

Fig. 10. AODV routing traffic received in mobile topologies.

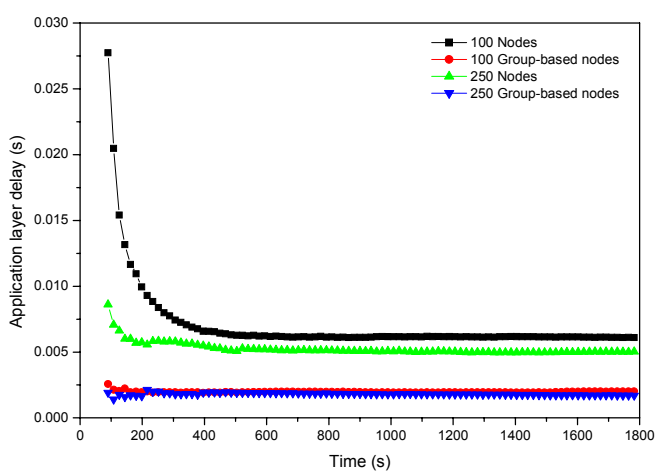

Fig. 12. OLSR average delay at the application layer in mobile topologies. 


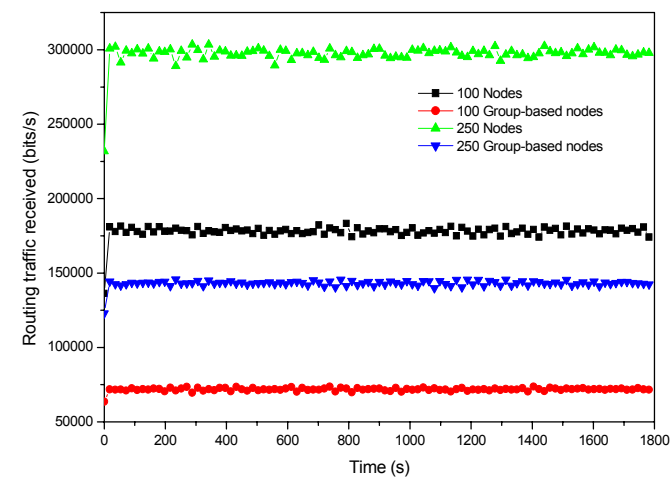

Fig. 13. OLSR routing traffic received in fixed topologies.

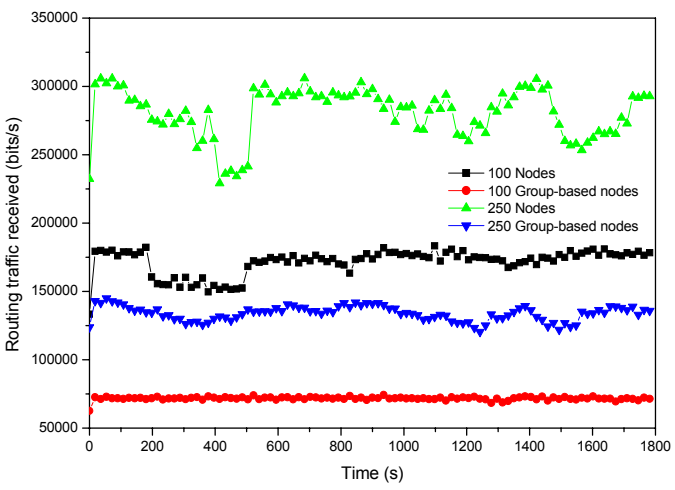

Fig. 14. OLSR routing traffic received in mobile topologies.

\section{Group-based topologies comparison}

In order to make the comparison of DSR, AODV and OLSR using group-based topologies, we have used the same test bench used in section 2. This comparison will show us which mobile and ad-hoc routing protocol have better features for groupbased topologies.

The average delay at MAC layer in fixed group-based topologies is shown in figure 15. All routing protocols have an average delay lower than 0.001 seconds when the network has converged in both 100-nodes and 250-nodes topologies. It shows that group-based topologies have a good behaviour. DSR protocol with 100 -node topology has been the one with worst behaviour and OLSR in 250-node topology has been the best one. OLSR protocol has the same delay (around 0.001 seconds) for both topologies, 100-nodes and 250-nodes, approximately, and it is the most stable. Figure 16 shows the simulation for mobile and errors and failures topologies. All protocols have a delay lower than 0.001 seconds when the network has converged. In this case, AODV protocol has the worst behaviour and OLSR protocol is the most stable.

When the average throughput consumed in the fixed group-based topologies is compared (Figure 17), the protocol that consumes lowest throughput is the DSR protocol $(90 \mathrm{Kbits} / \mathrm{s}$ in the 100 -node topology and $170 \mathrm{Kbits} / \mathrm{s}$ in the 250 -node topology). The protocol with the most stable throughput consumed is the OLSR protocol. When the network converges, both AODV and OLSR protocols have the same average throughput in the 100-nodes topology, but the OLSR protocol has the lowest convergence time. In case of having a group-based topology with mobility and errors and failures (see figure 18), the results are very similar to the previous ones. The protocol that consumes lower throughput is DSR. AODV protocol consumes lower throughput while the network is converging, but this throughput becomes very similar to the one given by OLSR protocol when the network converges. OLSR protocol is still the most stable. 
Then, we analyzed the protocols behaviour when there is MANET traffic. In fixed group-based topologies (see figure 19), the 250-nodes topology shows that the protocol with lower traffic is AODV (40 bits/s approximately) and the one with highest traffic is OLSR. In the 100-nodes topology all protocols have similar behaviour (between $160 \mathrm{bit} / \mathrm{s}$ and $180 \mathrm{bits} / \mathrm{s}$ ). When the network has converged, we can consider AODV and DSR as the best ones and OLSR as the worst. When there is mobility in the group-based topology (see figure 20), the protocol with lowest MANET traffic in the 250 -nodes topology is DSR protocol ( 80 bits/s approximately) and the worst is OLSR protocol. In the case of 100-node topology the one with lowest MANET traffic is DSR protocol and the worst OLSR.

When we analyze the routing traffic sent in fixed group-based topologies (see figure 21) we observe that the one which sends more routing traffic is AODV protocol, (around $120 \mathrm{Kbit} / \mathrm{s}$ in the 250-nodes group-based topology and $56 \mathrm{Kbits} / \mathrm{s}$ in the 100-nodes group-based topology). OLSR protocol has the best behaviour. It is more stable than the other ones and it sends lower routing traffic than the others (64 $\mathrm{Kbits} / \mathrm{s}$ in case of the 250 -nodes topology and $28 \mathrm{Kbits} / \mathrm{s}$ in the 100-nodes topology). When we analyze the mobile group-based topology (figure 22), although the routing traffic has decreased very few, the behaviour of the protocols is very similar to the fixed group-based topologies (Figure 21). AODV is the worst protocol because it is the one which sends more routing traffic to the network and OLSR is the most stable and the one which sends lower routing traffic to the network. The one which has worst stability in mobile group-based topologies is the DSR protocol.

The routing traffic sent is obtained by measuring every node as a source and figures 21 and 22 give the whole routing traffic sent by all of them. However, the routing traffic received is obtained by adding the traffic received by all nodes. The routing traffic received in fixed and mobile group-based topologies is shown in figures 23 and 24 respectively. We can see that it is more than the double of the values obtained for the routing traffic sent. In fixed group-based topologies (see Figure 23) AODV protocol is the one that gives higher routing traffic received (around $250 \mathrm{Kbit} / \mathrm{s}$ in 250-nodes topology and $135 \mathrm{Kbits} / \mathrm{s}$ in 100-nodes topology). OLSR protocol is the most stable and the one with lower routing traffic received (145 $\mathrm{Kbits} / \mathrm{s}$ in 250 -nodes topology and $70 \mathrm{Kbits} / \mathrm{s}$ in 100 -nodes topology). When the mobile group-based topologies are analyzed (figure 24), AODV protocol is the one that has worst behaviour and OLSR is the most stable and the one that has lower routing traffic sent. DSR protocol is the most instable.

Figure 25 shows the average delay at application layer in fixed group-based topologies. The protocol most instable and with higher delay in 100-nodes and 250nodes topologies is AODV protocol. It has peaks with more than 0.45 seconds and it is stabilized around 1700 seconds with a mean value of 0.15 seconds. DSR and OLSR are the ones with lowest delay. Figure 26 shows the average delay at application layer in mobile group-based topologies. DSR protocol is the one that has worst delay till the network converges. Then, when the network is stabilized, the worst is AODV protocol which has delays between 0.1 and 0.15 seconds. OLSR protocol gives the lowest delays.

Then, we have compared DSR and AODV in some common reactive protocols features. In figure 27 the average number of hops in a path for fixed group-based topologies can be observed. DSR protocol has an average value of hops close to 5 in 
the 250-nodes topology when the network has converged. The number of hops in the 100 -nodes topology is slightly lower. AODV has lower average number of hops (around 3.25 hops in the 250-nodes case and 2.75 in the 100-nodes case). The convergence time for the DSR protocol is quite lower than AODV, but it is more instable. In the case of mobile group-based topologies (see figure 28) the behaviour is similar as the previous one, so there is not any dependence on the mobility.

Now we have analyzed the route request sent in reactive protocols for fixed and group-based topologies (figures 29 and 20 respectively). AODV protocol is the one with most number of route requests sent (860 approximately in 250-nodes topology and 330 approximately in 100-nodes topology). We have observed a relationship between the number of route requests sent in the AODV protocol and number of nodes in the topology. There is approximately a factor of 3.3. In the DSR protocol, the number of route requests sent is equal to 730 in the 250-nodes topology and 190 in the 100-nodes topology. Both, fixed and mobile, present the same behaviour. We have observed that the route request sent is the only parameter that gives worst values in group-based topologies than in regular topologies.

Table 1 shows the best and worst protocols for all parameters analyzed.

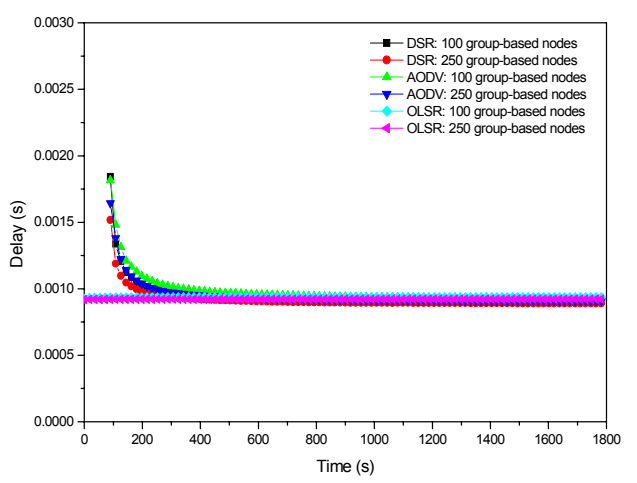

Fig. 15. Comparison of average delays at MAC layer in fixed topologies.

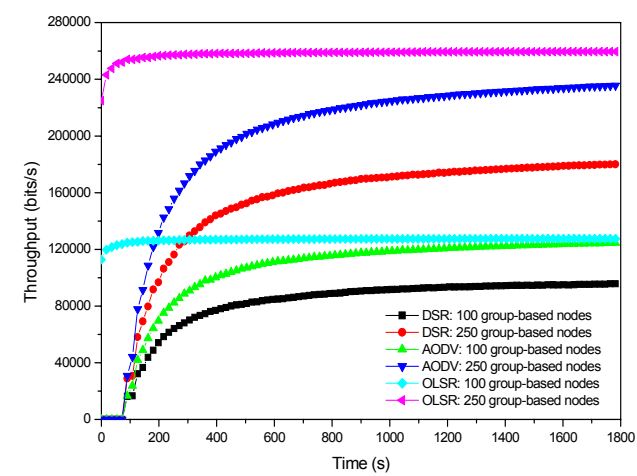

Fig. 17. Comparison of average throughputs consumed in fixed topologies.

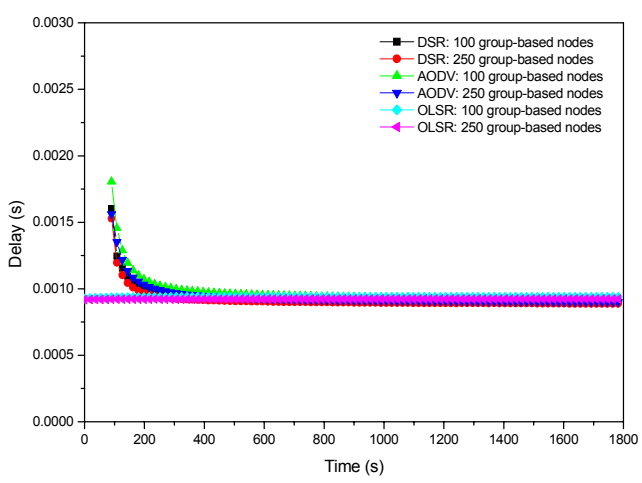

Fig. 16. Comparison of average delays at MAC layer in mobile topologies.

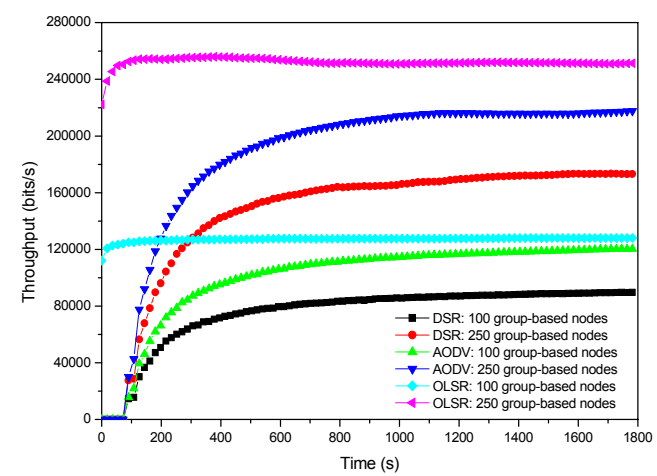

Fig. 18. Comparison of average throughputs consumed in mobile topologies. 


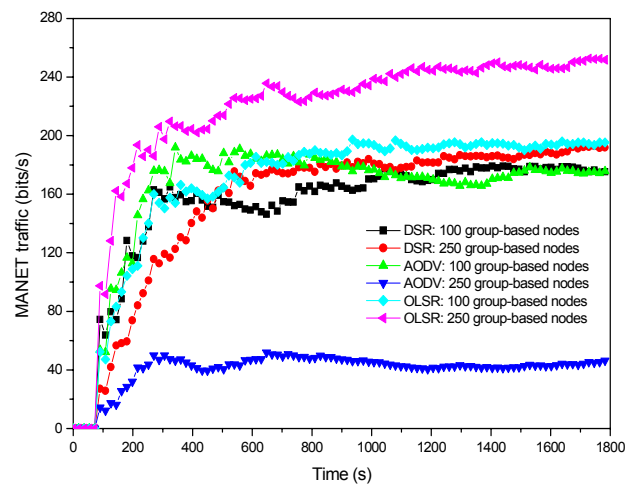

Fig. 19. Comparison of average MANET traffic in fixed topologies.

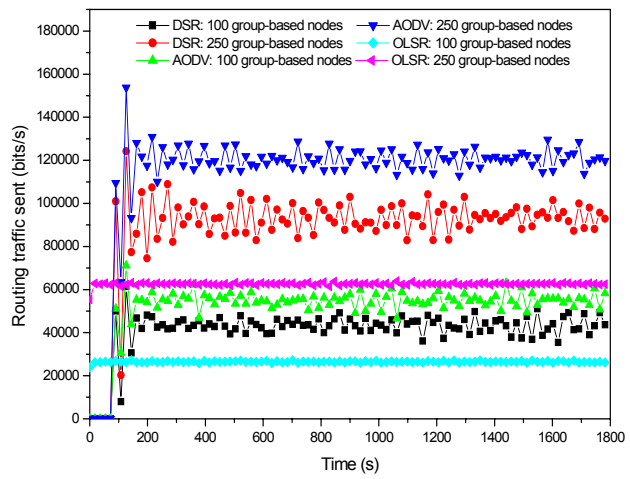

Fig. 21. Comparison of routing traffic sent in fixed topologies.

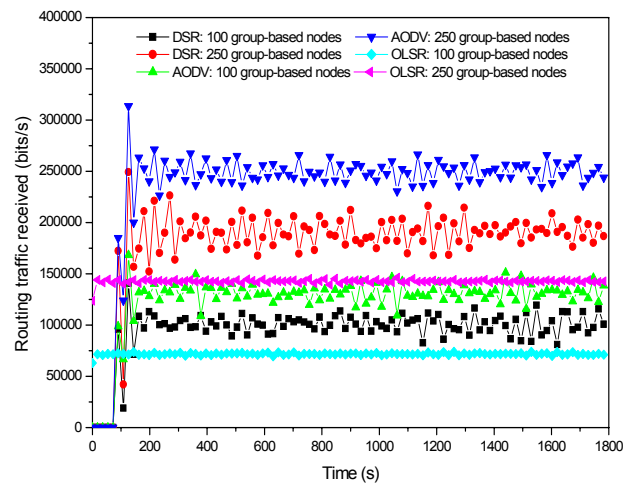

Fig. 23. Comparison of routing traffic received in fixed topologies.

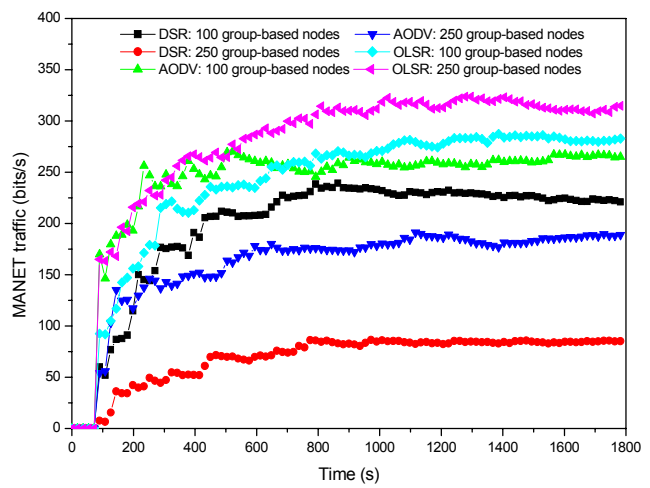

Fig. 20. Comparison of average MANET traffic in mobile topologies.

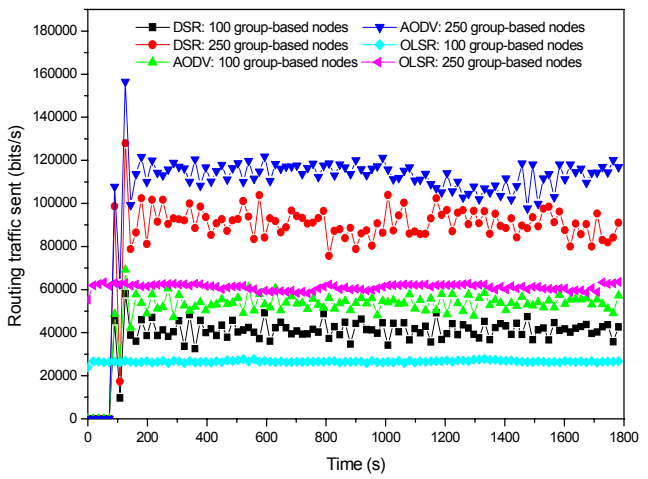

Fig. 22. Comparison of routing traffic sent in mobile topologies.

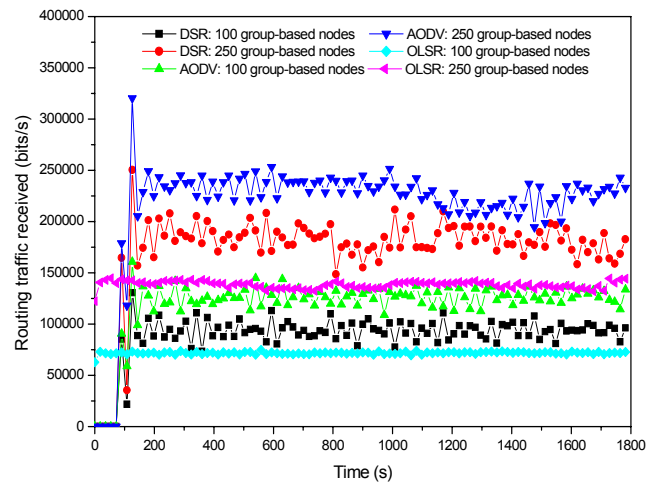

Fig. 24. Comparison of routing traffic received in mobile topologies. 


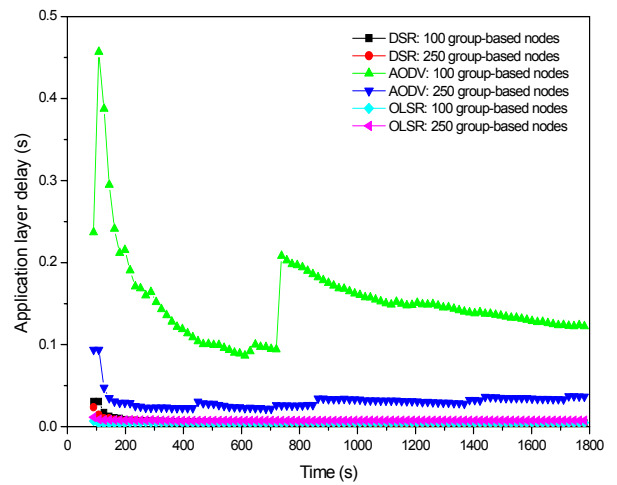

Fig. 25. Comparison of the average delay at application layer in fixed topologies.

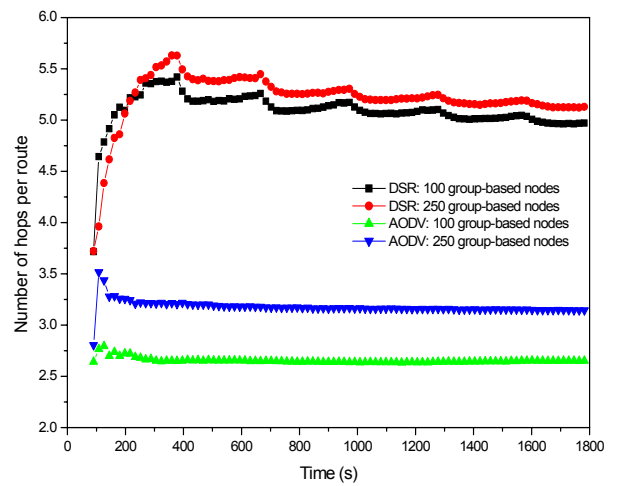

Fig. 27. Comparison of the average number of hops in a path in fixed topologies.

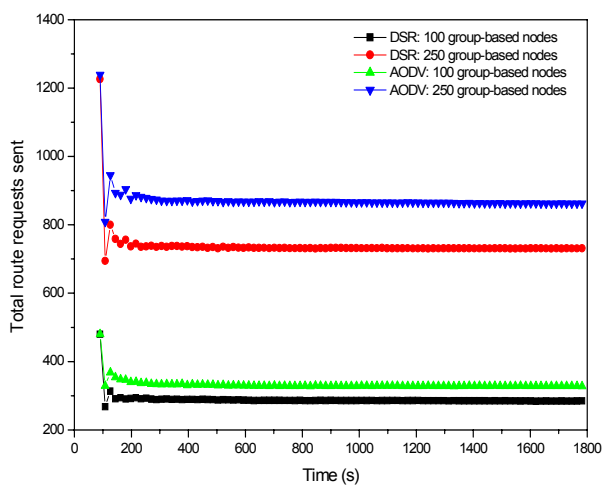

Fig. 29. Comparison of the average number of route requests sent in fixed topologies.

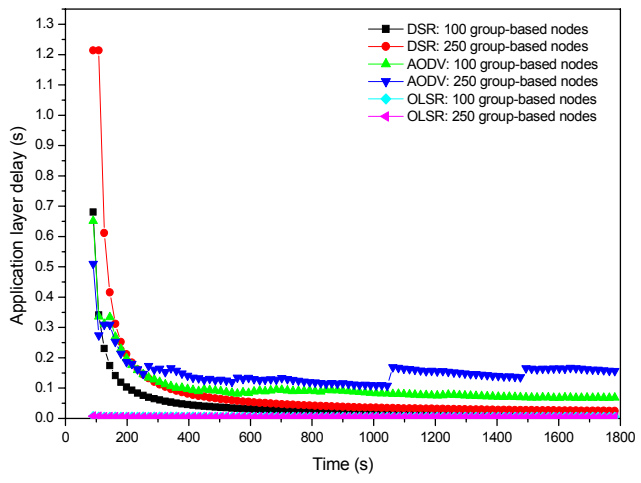

Fig. 26. Comparison of the average delay at application layer in mobile topologies.

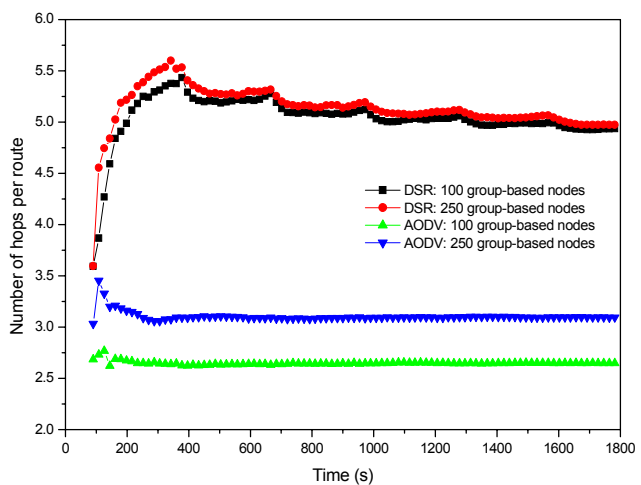

Fig. 28. Comparison of the average number of hops in a path in mobile topologies.

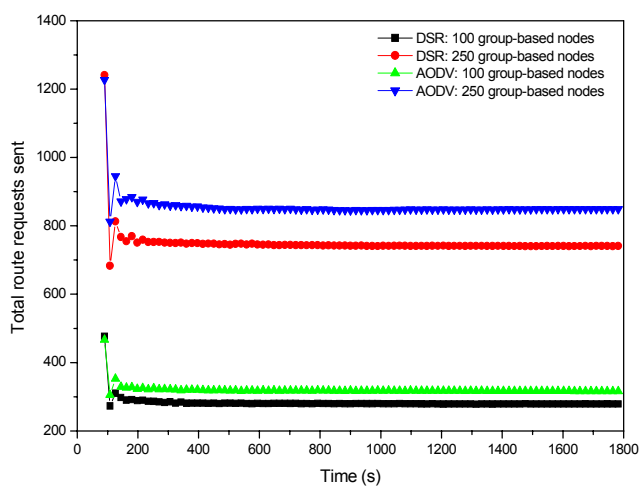

Fig. 30. Comparison of the average number of route requests sent in mobile topologies. 
Table 2. Comparison of mobile and ad-hoc routing protocols in group-based topologies.

\begin{tabular}{lcccc}
\hline & Best in fixed & Best in mobile & Worst in fixed & Worst in mobile \\
\hline Delay at MAC layer & OLSR & OLSR & DSR & AODV \\
Throughput consumed & DSR & DSR & AODV \& OLSR & AODV \& OLSR \\
MANET traffic & AODV & DSR & OLSR & OLSR \\
Routing traffic sent & OLSR & OLSR & AODV & AODV \\
Routing traffic received & OLSR & OLSR & AODV & AODV \\
Delay at application layer & DSR \& OLSR & OLSR & AODV & AODV \\
Average number of hops in a path & AODV & DSR & AODV & DSR \\
Route requests sent & DSR & AODV & DSR & AODV \\
\hline \hline
\end{tabular}

\section{Conclusions}

We have simulated 3 MANET routing protocols with grouping nodes, to demonstrate that group-based topologies improve the network performance. The best improvement percentage has been the DSR protocol when the average delay at the application layer has been simulated. We have observed more improvement in fixed topologies when there are 250 nodes in the topology, but when there is a mobile topology, the improvement is higher in the topology with 100 nodes. When a routing protocol is the best one in a fixed group-based topology, it continues being the best one in the mobile group-based topology. On the other hand, we have observed that a routing protocol, which is the best (or worst) in a group-based fixed topology, could not be the best (or worst) in the mobile topology. The routing protocol that has appeared more as the best one has been OLSR and the one that has appeared as the worst one has been AODV.

\section{References}

1. D. Johnson, Y. Hu, D. Maltz. "The Dynamic Source Routing Protocol (DSR) for Mobile Ad-hoc Networks for IPv4". RFC 4728. February, 2007.

2. C. Perkins, E. Belding-Royer and S. Das, "Ad Hoc On-Demand Distance Vector (AODV) Routing". RFC 3561. July, 2003.

3. T. Clausen and P. Jacquet. "Optimized Link State Routing Protocol". RFC 3626. Oct. 2003.

4. R. Misra, C. R. Mandal, "Performance comparison of AODV/DSR on-demand routing protocols for ad hoc networks in constrained situation", IEEE International Conference on Personal Wireless Communications, 2005. Pp. 86- 89. January 2005

5. G. Jayakumar, G. Ganapathy "Performance Comparison of Mobile ad-hoc Network Routing Protocol". Int. Journal of Computer Science and Network Security, Vol.7 No.11, Nov. 2007.

6. H. Jiang and J. J. Garcia-Luna-Aceves, "Performance comparison of three routing protocols for ad hoc networks". Proceedings of IEEE ICCCN 2001.

7. S. Ahmed, M. S. Alam, "Performance Evaluation of Important Ad Hoc Network Protocols". EURASIP Journal on Wireless Communications and Networking. Vol. 2006. Pages 1-11.

8. P. Johansson, T. Larsson, N. Hedman, B. Mielczarek, and M. Degermark, "Scenario-Based Performance Analysis of Routing Protocols for Mobile Ad Hoc Networks". Proc. of the 5th ACM/IEEE int. conf. on Mobile computing and networking. Pp. 195-206. 1999.

9. J. Broch, D. A. Maltz, D. B. Johnson, Y. Hu, J. Jetcheva, "A Performance Comparison of Multi-Hop Wireless Ad Hoc Network Routing Protocols". Proc. of the $4^{\text {th }}$ ACM/IEEE int. conf. on Mobile computing and networking. Pp. 85-97. 1998.

10. Azzedine Boukerche, "Performance Evaluation of Routing Protocols for Ad Hoc Wireless Networks", Journal of Mobile Networks and Applications. Vol. 9, No 4. Pp. 333-342, 2004.

11. OPNET Modeler website. At, http://www.opnet.com/solutions/network_rd/modeler.html 